\title{
REVISIÓN
}

\section{Respuestas ecofisiológicas de plantas en ecosistemas de zonas con clima mediterráneo y ambientes de altamontaña}

\author{
Ecophysiological responses of plants in ecosystems with Mediterranean-like climate \\ and high mountain environments
}

\section{H. MARINO CABRERA}

Instituto de Biología (Botánica), Facultad de Ciencias Básicas y Matemáticas, Universidad Católica de Valparaíso, Casa Central, Avenida Brasil 2950, Valparaíso, Chile; e-mail: hcabrera@ucv.cl

\begin{abstract}
RESUMEN
Esta es una revisión de los estudios en ecofisiología en plantas de zonas con clima tipo mediterráneo, con enfásis en la fotosíntesis frente a los múltiples estrés de estos ambientes. Se hace un acercamiento ecofisiológico al estudio de la distribución de las formas de vida y de las especies, particularmente en la zona central de Chile. Se analiza el efecto del estrés hídrico (sequía), térmico (temperaturas) y lumínico (radiación) en la fotosíntesis. El estrés hídrico sería un factor determinante en la distribución de árboles siempreverdes y semideciduos en altitudes bajas, mientras que en altitudes intermedias (en el límite arbóreo) o mayores, las temperaturas y/o el estrés hídrico junto con el estrés lumínico afectarían a arbustos y cojines. Se discutirá el fenómeno de la fotoinhibición de la fotosíntesis causada por los múltiples estrés que enfrentarían las plantas en zonas de clima tipo mediterráneo, explicando los conceptos teóricos básicos de la emisión de fluorescencia de la clorofila a y la fotoprotección otorgada por las xantofilas. Se proponen hipótesis para explicar como estos múltiples estrés modulan la distribución y los patrones fenológicos estacionales e interanuales en plantas. Se comparan especies filogenéticamente cercanas (e.g., congenéricas) y con diferencias interespecíficas en los caracteres fenotípicos que se correlacionan con parámetros del ambiente, que se explican mediante procesos adaptativos y que no son producto de la inercia filogenética.
\end{abstract}

Palabras clave: ecofisiología, fotosíntesis, estrés hídrico, térmico y lumínico.

\begin{abstract}
This review highlights the studies on plant physiological ecology of Mediterranean-like climate zones, with interest in the photosynthesis and in the multiple stress characteristics of these environments. It incorporates an eco-physiological approach to the study of the distribution of the life forms and species of Mediterranean ecosystems, particularly in the Mediterranean zone of central Chile. It is emphasized the effect of drought (water), thermal (temperatures) and luminic stress (radiation) on photosynthesis. Drought stress would be the determinant factor in the distribution in evergreen and deciduous trees at low altitudes, whereas in shrubs and cushions at intermediate altitudes -at the treeline- or higher, it would be the temperatures and/or the combination of both stresses, moreover the light stress. The photoinhibition of photosynthesis caused by the multiple stresses in Mediterranean-climate zones is also discussed, explaining the theoretical basic concepts in chlorophyll "a" fluorescence and photoprotection provided by the xanthophylls cycle. The hypotheses to explain how these multiple stresses modulate the distribution and the seasonal and annual phenological patterns of plants are postulated. Finally, in order to avoid the clear ecological differences in the ecophysiological responses of plants, comparisons are done among phylogenetically close species (i.e., congener) and interspecific differences in phenotypic characters that are correlated with environmental parameters. These aspects are explained through examing the adaptative processes and not throughout the independence of the characteristics resulting from the phylogenetic inertia.
\end{abstract}

Key words: ecophysiology, photosynthesis, water, temperature and light stress.

\section{INTRODUCCIÓN}

El objetivo de esta revisión es discutir los estudios en ecofisiología de plantas en ecosistemas con clima tipo mediterráneo, con énfasis en la fotosíntesis frente a los múltiples estrés característicos de estos ambientes. Con este propósito, se incorpora un acercamiento ecofisiológico al estudio de la distribución de las formas de vida y de las especies en los ecosistemas mediterráneos, 
particularmente en Chile central. Se destaca el papel en la fotosíntesis del estrés hídrico (sequía), térmico (temperaturas) y lumínico (radiación). En las altitudes bajas, el estrés hídrico sería un factor determinante en la distribución de árboles siempreverdes y semideciduos, mientras que en las altitudes intermedias -en el límite arbóreo- o mayores, en los arbustos y cojines, lo serían las temperaturas y/o la combinación de ambos tipos de estrés. En esta revisión se comparan investigaciones entre especies filogenéticamente cercanas (e.g., congenéricas) y caracteres fenotípicos que se correlacionan con parámetros del ambiente, que logren explicarse mediante procesos adaptativos y no debido a la inercia filogenética.

\section{RESPUESTAS ADAPTATIVAS DE PLANTAS EN} ECOSISTEMAS DE ZONAS CON CLIMA TIPO MEDITERRÁNEO

Las variables climáticas definen las áreas de distribución, los límites de sobrevivencia y los pisos altitudinales de vegetación e influyen, a una escala menor, en la distribución de especies y comunidades (Nahal 1981, Woodward \& Williams 1987, Prentice et al. 1992). Los ecosistemas mediterráneos son ejemplos clásicos de convergencia (Mooney \& Dunn 1970, di Castri 1973, 1990, Pearson 1976, Mooney et al. 1977, Arroyo et al. 1995, Cowling et al. 1996) en que son semejantes múltiples caracteres ecomorfológicos de las especies (Montenegro \& Ginocchio 1995) apoyando la hipótesis que bajo regímenes climáticos similares, las respuestas adaptativas de las plantas son limitadas, dando como resultado ecosistemas estructural y funcionalmente semejantes (Mooney \& Dunn 1970, Mooney et al. 1977, Cody \& Mooney 1978). En los ecosistemas de zonas con clima tipo mediterráneo, se han descrito diversos tipos de estrés que restringirían las oportunidades de desarrollo y sobrevivencia de las especies vegetales. Ejemplo de esto es la zona central de Chile, que abarca un amplio rango altitudinal (Arroyo et al. 1995) y se caracteriza por una disponibilidad hídrica limitada, así como por diferencias notables, diurnas o estacionales, en las temperaturas (Santibáñez \& Uribe 1992). Estos factores determinan que el crecimiento y la reproducción estén restringidos a las estaciones de primavera y verano (Mooney et al. 1977), particularmente en las zonas con clima de alta montaña (Arroyo et al. 1981). La distribución altitudinal de formas de vida y patrones fenológicos de las especies reflejarían la interacción de estos dos factores (Mooney et al.
1977, Armesto et al. 1979, Hoffmann \& Walker 1980, Arroyo et al. 1981, Arroyo et al. 1982, Hoffmann \& Hoffmann 1982, Montenegro 1987, Pereira \& Chávez 1993, Arroyo et al. 1995, Lamont 1995).

\section{ECOFISIOLOGÍA EN PLANTAS EN ECOSISTEMAS DE ZONAS CON CLIMA TIPO MEDITERRÁNEO}

En los ecosistemas mediterráneos, la vegetación, las especies y las formas de vida se hallan distribuidas en un gradiente de aridez: las plantas suculentas ocupando los sitios más xéricos, los arbustos de hoja pequeña, deciduos y/o siempreverdes esclerófilos de pequeño tamaño dominantes hacia los extremos xéricos a baja altitud, las especies de arbustos siempreverdes en los sitios más mésicos a baja elevación y los arbustos de hojas mesofíticas, deciduas de invierno, en los hábitats húmedos a mayor altitud (Mooney \& Kummerow 1971, Mooney et al. 1977). En parte, esta distribución se ha explicado por respuestas adaptativas de las plantas a la disponibilidad de agua (Armesto \& Martínez 1978, Martínez \& Armesto 1983).

En arbustos siempreverdes, los cursos de estrés hídrico a lo largo del año estarían relacionados a sus hábitos de enraizamiento: las especies con raíz profunda, tienen potenciales hídricos del xilema altos y menos variables, en contraste con las especies de sistemas radicales más superficiales -con mayores y más variables niveles de estrés(Giliberto \& Estay 1978). Las especies siempreverdes, presentan una variación estacional menor que las deciduas al finalizar el período de sequía -sin síntomas de sequía y/o con estrés hídrico por un corto período- manteniendo altas tasas de transpiración gracias al agua disponible en los perfiles profundos del suelo (Canadell \& Zedler 1995). La importancia de esto para especies mediterráneas siempreverdes, está en que permite un balance de carbono positivo, principalmente en la época seca -cuando el estado hídrico de las plantas podría limitar las tasas fotosintéticas- tal como fué descrito en Heteromeles arbutifolia, un arbusto siempreverde de California (Mooney \& Chu 1974). Igual comportamiento descrito en Chile en Quillaja saponaria y Lithrea caustica, contrasta con el que presenta Cryptocaria alba, con un balance negativo debido a que la fotosíntesis es muy baja en el nivel de estrés hídrico encontrado en esta especie (Martínez $\&$ Armesto 1983) presumiblemente como consecuencia de su raíz superficial (Riveros et al. 1976). Esta limitación hace difícil que esta especie alcance el agua disponible en los niveles profundos del suelo durante la estación seca e igualmente, 
explica las tasas fotosintéticas de verano en $Q$. saponaria y L. caustica (Martínez \& Armesto 1983) con raíces que llegan a profundidades de 78 y 3-4 m, respectivamente (Giliberto \& Estay 1978). Los patrones de enraizamiento en plantas -como otros aspectos de su morfología- resultan de la influencia del ambiente en el desarrollo de un plan genético básico y en este caso los factores primarios que controlan estos patrones son la humedad, los nutrientes y las propiedades físicas del suelo (Canadell \& Zedler 1995) parámetros que cambian tanto en una escala espacial amplia como local (Kummerow 1981). Estas estrategias permiten a las especies siempreverdes con raíces profundas, utilizar a lo largo del año la humedad disponible en los horizontes más bajos y complementariamente, con un sistema menos profundo, acceder al mayor contenido de nutrientes en suelos superficiales y usar el agua de lluvia cuando los horizontes más profundos no están totalmente recargados después del verano (Canadell \& Zedler 1995).

Muchas especies deciduas en las regiones mediterráneas presentan un sistema radicular superficial (Mooney \& Dunn 1970) ocupando los sitios mas xéricos de disponibilidad de agua y haciéndose dormantes frente a la sequía (Canadell \& Zedler 1995). En arbustos deciduos, evasores de la sequía y considerados mejor adaptados para enfrentar prolongados períodos de déficit de agua, la estrategia adaptativa común es la reducción del área foliar, principalmente por la pérdida estacional de las hojas (Pereira \& Chávez 1993). Estas respuestas adaptativas al estrés hídrico en plantas esclerófilas siempreverdes y deciduas, las dos formas de vida mayoritariamente representadas por debajo del límite altitudinal de los árboles en la vegetación de tipo mediterráneo, han sido señaladas para explicar los patrones de distribución de las especies y de los tipos de vegetación en estos ecosistemas (Mooney \& Dunn 1970, Mooney \& Kummerow 1971, Parsons \& Moldenke 1975, Armesto \& Martínez 1978, Rundel 1981, Nardini et al. 1999, Squeo et al. 1999, Verdaguer et al. 2000).

La coexistencia de plantas ha sido también relacionada con diferencias entre las especies en características tales como fenología, requerimientos nutricionales y la distribución de las raíces con la profundidad (Al-Mufti et al. 1977, Berendse 1982). Estas diferencias tienden a reducir la competencia entre las especies, permitiendo la coexistencia de plantas, como ha sido descrito para dos especies mediterráneas de Quercus (Nardini et al. 1999). Muchas especies de plantas difieren en el tiempo y la profundidad del suelo al cual su sistema radicular está activo y en especies coexistentes, estas diferencias podrían ser importantes debido a la competencia entre ellas. Las especies más competitivas estarían activas en las capas superficiales más fértiles del suelo, obligando de esta forma a las especies menos competitivas a explotar capas del suelo más profundas (Berendse 1981, 1982). En este sentido, los gradientes ambientales son importantes para estudiar la relación entre la competencia y los factores del ambiente, principalmente en ecosistemas en que el agua limita fuertemente el crecimiento de las plantas y en que el suelo frecuentemente se encuentra desnudo o la vegetación se halla dispersa (Pantastico-Caldas \& Venable 1993, Hook et al. 1994, Belcher et al. 1995, Nardini et al. 1999, Squeo et al. 1999, Coomes \& Grubb 2000).

\section{EFECTO DEL ESTRÉS HÍDRICO SOBRE LA FOTOSÍNTESIS}

Con un incremento de la sequía, las comunidades siempreverdes dominadas por árboles son reemplazadas por arbustos y posteriormente, por arbustos deciduos, los primeros con una capacidad fotosintética menor en comparación a las tasas en los deciduos (Mooney \& Dunn 1970). En estos últimos, el sistema fotosintético no presentaría mecanismos importantes para controlar la pérdida de agua, con mayores tasas transpiratorias y niveles de estrés hídrico más tempranos que en los siempreverdes (Canadell \& Zedler 1995), que tienen una densidad de hojas mayor, importante en la conservación de agua, en la duración y en la protección a la predación foliar (Mooney \& Dunn 1970, Mooney et al. 1977). Se ha sugerido para los elementos siempreverdes, que tanto el desplazamiento competitivo por la luz en los ambientes más mésicos y frente a la sequía, la disminución estacional de la ganancia potencial de carbono, favorecerían que los elementos deciduos se encuentren en hábitats más secos (Mooney \& Dunn 1970, Armesto \& Martínez 1978, Nardini et al. 1999). Un análisis en la capacidad fotosintética en especies esclerófilas siempreverdes de California (Mooney et al. 1975) y en árboles de Portugal (Faria et al. 1998) sugiere que la disponibilidad de agua y el cierre estomático es la limitación primaria y la explicación principal en la ganancia potencial de carbono. Estudios en diferentes especies de Quercus, sugieren que no habría diferencias en la eficiencia intrínseca del uso del agua entre las especies de árboles decíduos ( $Q$. pubescens) y siempreverdes ( $Q$. ilex) que coexisten en sitios con diferencias en disponibilidad hídrica (Damesin et al. 1997). En Q. ilex, el déficit hídrico estacional por la sequía de verano 
es importante en las plantas por el efecto sobre las relaciones hídricas, la transpiración y el comportamiento estomático (Tognetti et al. 1998). En diferentes especies se han descrito los cambios tanto en la fotosíntesis como en la conductancia de los estomas en relación a una condición más cálida y seca producto del cambio climático global (Penuelas et al. 1998) sugiriéndose un ajuste hacia abajo en la capacidad fotosintética frente a una mayor concentración de $\mathrm{CO}_{2}$ (Tognetti et al. 2000).

Encelia canescens, una especie decidua de sequía, posee mayores ángulos foliares en condiciones de mayor falta de agua -como una estrategia para reducir la carga energética y la tasa de transpiración sin un cierre estomático- lo que le permite mantener altas tasas de fotosíntesis (Squeo et al. 1994). En las especies deciduas, se ha sugerido que la capacidad para ocupar un rango más grande de distribución, estaría relacionada a su habilidad para cambiar su morfología (tamaño) y la duración de sus actividades estacionales (fenologías) con respuestas especie-específicas (Hoffmann \& Walker 1980). En Chile, hasta ahora no se ha evaluado la estacionalidad de las hojas, siempreverdes y deciduas, con requerimientos específicos y limitaciones estacionales de la disponibilidad del agua en la actividad fotosintética a lo largo del año en un gradiente altitudinal. Para evaluar estas respuestas en las plantas, como estrategias para mantener y optimizar el balance de agua y de carbono frente a la sequía estacional (Mooney 1983, Nardini et al. 1999) se deben caracterizar parámetros hídricos y fotosintéticos, estacionalmente y a lo largo del año, en especies congenéricas: una siempreverde (e.g., Kageneckia oblonga) y otra semidecidua (e.g., K. angustifolia). Estos árboles, endémicos de la zona mediterránea de Chile, están distribuidos ocupando ambientes diferentes, desde el nivel del mar hasta los ca. $1.800 \mathrm{~m}$ y desde los ca. 1.300 hasta los ca. $2.200 \mathrm{~m}$ de altitud, respectivamente. Ya que los rangos de su distribución altitudinal se superponen, esto permitiría explicar los factores ambientales que separan la distribución de estas especies entre los pisos altitudinales de la vegetación (ca. $1.600 \mathrm{~m}$ ) en que abruptamente cambia la proporción de las formas de vida (Hoffmann \& Hoffmann 1982, Arroyo \& Uslar 1993). Además, debido a que el género alcanza el límite de la vegetación arbórea (Arroyo et al. 1981), se comprendería la interacción de los factores de estrés en limitar la distribución de la forma de vida arborescente hacia altitudes mayores. Esto ocurre para $K$. angustifolia en los $2.200 \mathrm{~m}$, altitudinalmente más abajo al compararlo con el límite arbóreo descrito en otros ecosistemas (Cabrera 1996, Rada et al. 1996, Cavieres et al. 2000).

\section{EFECTOS DEL ESTRÉS TÉRMICO SOBRE LA FOTOSÍNTESIS}

La estacionalidad de las lluvias y las temperaturas afectan la fotosíntesis en las especies. A pesar de que los patrones fenológicos son claras adaptaciones que permiten el crecimiento vegetativo en primavera y verano (Arroyo et al. 1981, Olivares \& Squeo 1999) cuando la humedad del suelo es abundante y las temperaturas permanecen favorables para la fotosíntesis, son escasos los estudios ecofisiológicos en ambientes de alta montaña con déficit hídrico y baja temperatura (Chabot \& Billings 1972). Además, en plantas de alta montaña no se han realizado investigaciones para estimar el efecto de la altitud en el potencial fotosintético de fijación del $\mathrm{CO}_{2}$ (Smith \& Donahue 1991) y/o el impacto de temperaturas no congelantes en la fotosíntesis de las plantas en estos ambientes (Allen \& Donald 2001). Los estudios fenológicos y reproductivos de la vegetación andina en Chile central (Arroyo et al. 1981, Arroyo et al. 1982, Arroyo et al. 1995) desconoce la relación entre las temperaturas, la disponibilidad hídrica estacional y las respuestas adaptativas de las plantas. Excepto por los trabajos de Squeo et al. (1996) y Rada et al. (1999), no se han realizado otros trabajos de investigación sobre los mecanismos de tolerancia o evasión tanto a la sequía y/o a las temperaturas bajas en especies de alta montaña. Así mismo, tampoco se ha considerado al déficit hídrico estacional y la mayor sensibilidad en la vegetación a las temperaturas bajas, como factores principales en limitar los rangos de distribución altitudinal de las especies: En Chile central, los árboles y arbustos están restringidos a elevaciones por debajo de $2.600 \mathrm{~m}$ y los cojines son dominantes en la vegetación por arriba de los 2.600-2.700 m.

En las plantas de alta montaña, principalmente por arriba del límite arbóreo, se presentarían otras limitantes. En plantas bajo sequía y/o temperaturas bajas, debido a las tensiones negativas en el xilema, los tallos se hacen más sensibles a cavitación (Grace 1993). Respecto al embolismo inducido por estrés hídrico, las evidencias sugieren que la formación de gas y el rompimiento de la columna de agua en los tallos no estaría en función directa con el tamaño de los conductos, sino más bien con el máximo tamaño del poro en las paredes celulares de vasos y traqueidas (Sperry \& Tyrre 1988, Jarbeau et al. 1995). En contraste, el embolismo inducido por congelamiento está 
fuertemente relacionado con el diámetro o volumen de los conductos xilemáticos (Sperry \& Sullivan 1992, LoGullo \& Salleo 1993). El significado ecológico y evolutivo del embolismo en el xilema por estrés hídrico y/o por congelamiento, aún permanece poco claro (Sperry \& Tyree 1988) así como el mecanismo de la formación de gas por embolismo en tallos leñosos de las plantas vasculares, que parece depender del tipo de estrés ambiental (Wang et al. 1992, Kolb \& Davis 1994, Redtfeldt \& Davis 1996, Sperry 1995). Ambos factores son capaces de inducir embolismo en los tallos, potenciándose sinérgica y estacionalmente (Langan et al. 1997). Se destaca el papel de la fotoprotección en las condiciones de invierno (García-Plazaola et al. 1999a) así como las diferencias en la vulnerabilidad y la respuesta a sequía y/o congelamiento como factores limitantes en la distribución en juveniles de Quercus ilex a lo largo de un gradiente latitudinal (Nardini et al. 2000). En los géneros Pinus y Abies en la Sierra Nevada en California, se han descrito diferencias en el patrón de uso del agua en respuesta a la variación estacional del clima y se ha relacionado esto con el efecto sobre la distribución de las especies en un clima mediterráneo, inusual para bosques de coníferas (Royce \& Barbour 2001a, 2001b).

Respecto a los mecanismos adaptativos de tolerancia y evasión a temperaturas bajas, estos son considerados importantes en límites altitudinales de los ecosistemas y en las respuestas de las especies en altas montañas tropicales en Los Andes, Hawaii, África y subtropicales en Chile, con una relación entre la duración de las bajas temperaturas nocturnas y las formas de vida de las plantas. Las que crecen a nivel del suelo presentarían tolerancia, las arborescentes lo evaden (superenfrían) y las del estrato intermedio exhiben ambos mecanismos (Cabrera 1996). Excepto por el trabajo de Hoffmann \& Walker (1980), respecto a que la baja temperatura y una prolongada cobertura de nieve constituirían severos limitantes para la sobrevivencia de la vegetación a mayor altitud, se desconoce la tolerancia al frío en las especies altoandinas con distintas formas de vida en Chile central y como ambos factores en el invierno determinan en este ecosistema los limites altitudinales de la distribución de las especies y de las formas de vida. Es fundamental que las plantas presenten un balance de carbono positivo en ambientes con temperaturas bajas (Körner \& Larcher 1988) lo que explicaría porque Polylepis crece hasta los $4.600 \mathrm{~m}$, en que junto al eficiente mecanismo de resistencia a las temperaturas, permite ser el único árbol por sobre la línea del bosque continuo en Los Andes (Rada et al.
1996, Cavieres et al. 2000, Rada et al. 2001). Para formas de vida más pequeñas, además de la resistencia al frío, en la capacidad para adaptarse a las condiciones térmicas a mayor altura (Chabot \& Billings 1972) es determinante la plasticidad en la fotosíntesis -en respuesta a la temperaturapara el éxito de las especies en diferentes ambientes y microclimas (Cabrera et al. 1998). En la alta montaña de Chile central, a diferencia de lo señalado en otros ecosistemas en que el factor limitante sería principalmente la temperatura (Körner \& Larcher 1988), una menor disponibilidad hídrica limitaría el desarrollo de las formas de vida arbustivas a mayor altitud, afectando principalmente el balance hídrico y de carbono.

ESTRÉS HÍDRICO, TÉRMICO Y LUMÍNICO: FOTOINHIBICIÓN, FOTOPROTECCIÓN Y FOTOSÍNTESIS

\section{Fotoinhibición y fotoprotección}

Muchas especies de la vegetación de alta montaña presentan una actividad vegetativa restringida a los meses de primavera y verano (e.g., Arroyo et al. 1981) y en altitudes menores, las especies siempreverdes e incluso deciduas, presentan la mayor actividad vegetativa previo a la pérdida de las hojas, con la mayor cantidad de hojas a lo largo del año (Mooney et al. 1977, Hoffmann \& Walker 1980, Montenegro 1987). En climas mediterráneos, es en el verano en que las plantas están sometidas a estrés hídrico, altas temperaturas, con una mayor frecuencia de días claros, con alta radiación solar (Nahal 1981, Santibáñez \& Uribe 1992). Esta combinación de factores de estrés puede resultar en una disminución en la productividad fotosintética (Long et al. 1994). En las plantas, altos niveles de radiación, provocan fotoinhibición de la fotosíntesis y destrucción fotooxidativa del aparato fotosintético (Osmond 1994) caracterizado por una reducción en la eficiencia de la utilización de la luz (Krause \& Weis 1991). La fotoinhibición es provocada por la pérdida del funcionamiento de los fotosistemas II (FSII) que se manifiesta como una disminución, transitoria o permanente, en la eficiencia cuántica de la fotosíntesis (mol de $\mathrm{CO}_{2}$ fijado por mol de fotones absorbido) en niveles de luz de baja intensidad (Osmond 1994). Esta disminución en la eficiencia de la fotosíntesis y en el funcionamiento de los FSII, podría estar o no, bajo luz saturante, acompañada por una disminución en la máxima capacidad fotosintética ( $\mathrm{A}_{\max }$ ), en que bajo diferentes estrés o inherentes limitaciones en la capacidad para utilizar altos niveles de radiación, las hojas absorben más energía lumínica de la utili- 
zada en la fotosíntesis (Osmond 1981). La fotoinhibición puede ser el resultado del "fotodaño" directo en los FSII y producto de la "fotoprotección" en la cual la excesiva energía de excitación es reorientada y disipada principalmente como calor, procesos que logran un balance entre la energía recibida por los FSII con la capacidad de éstos para utilizarla (Baker 1991, Demmig-Adams \& Adams 1992, Aro et al. 1993, Long et al. 1994, Osmond 1994).

La protección ocurre mayoritariamente por medio de un mecanismo donde interviene un gradiente de $\mathrm{pH}$ transtilacoidal y pigmentos del ciclo de la xantofila (Demmig-Adams 1990, Pfündel \& Bilger 1994) que actuarían, sinérgicamente, en la disipación del exceso de energía. A pesar de que el mecanismo preciso de fotoprotección no se conoce (Bilger \& Björkman 1994, Osmond 1994), se evitaría el daño fotoinhibitorio por exceso de luz, protegiendo así a los FS II (Eickmeier et al. 1993, Horton et al. 1994, Pfündel \& Bilger 1994, Dekker \& Van Grondelle 2000). En la disipación del exceso de energía desde los complejos antena colectores de luz de carotenoides-clorofilas de los FS I y FS II, se requiere una alta concentración de protones dentro del tilacoide y es dependiente de la presencia de componentes de-epoxidados del ciclo de la xantofila (Demmig-Adams et al. 1995). Estos pigmentos (viola, antera y zeaxantina) tendrían un papel clave en la fotoprotección (DemmigAdams 1990) debido a que en hojas bajo exceso de luz, la zeaxantina se acumula estimulándose su síntesis, formándose dentro de pocos minutos en respuesta a las condiciones de estrés lumínico, correlacionándose al incremento en la tasa constante de disipación de calor, como una vía para "descargar" el cloroplasto de la energía en exceso (Björkman \& Demmig-Adams 1994). Se ha sugerido que la concentración de los pigmentos del ciclo de la xantofila determinaría la máxima cantidad de zeaxantina que podría acumularse y así mismo, la máxima protección disponible a través de este proceso disipativo (Demmig-Adams \& Adams 1992). La mayor parte de la información del papel del ciclo de la xantofila en la fotoprotección ha sido obtenida del análisis de la fluorescencia de la clorofila a, la que corresponde a una pequeña parte de la energía de la luz capturada por los pigmentos fotosintéticos y es predominantemente emitida por los FSII (Krause \& Weis 1991). Esta fluorescencia es baja cuando la energía de excitación absorbida es efectivamente convertida en fotoquímica, es decir alto consumo o "quenching" fotoquímico (quenching-qP) y por otros procesos diferentes al quenching-qP, los llamados en conjunto consumo no-fotoquímico
(quenching-qN). En las hojas bajo alta radiación, se ha correlacionado el incremento en $\mathrm{qN}$ con la formación de zeaxantina, lo que ocurriría de manera lineal proporcionalmente en respuesta al nivel de luz (Demmig-Adams 1990, Demmig-Adams et al. 1996, Müller et al. 2001). La emisión de fluorescencia de la clorofila a se ha convertido en uno de los métodos más importantes para evaluar el comportamiento fotosintético (Seaton \& Walker 1990, Agati et al. 1995). La fluorescencia mínima, $\mathrm{F}_{0}$, es determinada cuando todos los centros de reacción están abiertos $\left(Q_{\mathrm{A}}\right.$ totalmente oxidados) y la fluorescencia máxima, $\mathrm{F}_{\mathrm{M}}$, cuando todos los centros están cerrados $\left(Q_{\mathrm{A}}\right.$ totalmente reducidos). La diferencia entre $\mathrm{F}_{0}$ y $\mathrm{F}_{\mathrm{M}}$ es llamada fluorescencia variable, $\mathrm{F}_{\mathrm{V}}$. La razón $\mathrm{F}_{\mathrm{v}} / \mathrm{F}_{\mathrm{M}}$ en promedio es de 0,8-0,83 en hojas no fotoinhibidas, siendo una medida de la máxima eficiencia cuántica potencial de los FSII (Krause \& Weis 1991). Así como $\mathrm{F}_{\mathrm{V}} / \mathrm{F}_{\mathrm{M}}$ es la eficiencia intrínseca de los FSII en hojas en oscuridad cuando todos los centros de los FSII están abiertos, $\mathrm{F}_{\mathrm{V}}{ }^{\prime} / \mathrm{F}_{\mathrm{M}}$ ' lo es durante iluminación bajo radiación fotosintéticamente activa. Una disminución en la razón de la fluorescencia variable y máxima de la clorofila $\left(\mathrm{F}_{\mathrm{V}} / \mathrm{F}_{\mathrm{M}}\right.$ o $\mathrm{F}_{\mathrm{V}}$ ' $/ \mathrm{F}_{\mathrm{M}}$ ') es frecuentemente utilizada para monitorear cambios en la eficiencia fotoquímica del FSII. Esta última también refleja la eficiencia con la cual la energía de excitación capturada por la antena colectora de luz es traspasada hacia los centros de reacción de los FSII (Demmig-Adams et al. 1995).

Diferentes tipos de estrés ambiental que afectan la eficiencia del FSII, producen una disminución característica de la razón $\mathrm{F}_{\mathrm{V}} / \mathrm{F}_{\mathrm{M}}$, la que es usada como una manera de medir la eficiencia fotoquímica de los FSII (Baker 1991, Krause \& Weis 1991). En forma similar a la fotoinhibición que ocurre en hojas bajo exceso de luz (disminución en $\mathrm{F}_{\mathrm{V}} / \mathrm{F}_{\mathrm{M}}$ ) es que la fotoprotección también disminuye la eficiencia cuántica de la fotosíntesis $\mathrm{y}$ son estos procesos preventivos en las antenas colectoras de luz los que también causan una disminución en $\mathrm{F}_{\mathrm{V}} / \mathrm{F}_{\mathrm{M}}$ y en $\mathrm{F}_{\mathrm{V}}$ / $/ \mathrm{F}_{\mathrm{M}}$, por períodos variables de tiempo. Esto ocurre porque el proceso de disipación de la energía de excitación, antes que alcance los centros de reacción del FSII, compite con el fotoquímico por la energía de excitación y por esto la fotoprotección es asociada con una disminución en la eficiencia fotosintética intrínseca de los FSII (Melis 1999).

\section{Fotosintesis}

En las especies, la susceptibilidad a la fotoinhibición de la fotosíntesis varia amplia- 
mente con la adaptación genética, el estado fisiológico y la historia de vida de las plantas y depende, además de la luz, de otras condiciones ambientales, en particular las temperaturas, la sequía o la deficiencia de $\mathrm{CO}_{2}$ (Aro et al. 1993). Una fotoinhibición crónica, en que la eficiencia fotosintética en la planta no se recupera, provoca una disminución a largo plazo de la fotosíntesis y el crecimiento, lo que podría estar generalmente relacionado a otro estrés ambiental, e.g., por sequía o temperaturas, siendo en este caso posible que ocurra incluso a intensidades de luz moderadas (Long et al. 1994). En plantas bajo sequía, la reducción en la fotosíntesis resulta de una baja disponibilidad de $\mathrm{CO}_{2}$ debido al cierre estomático, e incapacidad de disipar la radiación solar como calor latente, con lo que, consecuentemente, la temperatura foliar se incrementa. El estrés hídrico predispone a las hojas a sufrir fotoinhibición, debido a que en potenciales hídricos bajos, la fotosíntesis puede ser alterada por efectos no-estomáticos, principalmente por reducción en la actividad de los FSII y las reacciones de transferencia de electrones (Cornic 1994). Por lo tanto, una reducción intracelular de $\mathrm{CO}_{2}$ y una disfunción bioquímica del cloroplasto, podrían reducir la tasa de asimilación de $\mathrm{CO}_{2}$ y la eficiencia cuántica del transporte de electrones fotosintéticos (Baker 1993). En esto, la reducción en la conductancia estomática, frecuente en árboles esclerófilos durante el período más caluroso del día -incluso en ausencia de un significativo déficit hídrico- da como resultado una disminución de la fotosíntesis neta (Tenhunen et al. 1984). Aunque las causas de esta disminución no se comprenden y están involucrados mecanismos regulatorios a nivel del estoma y del cloroplasto, tasas de fotosíntesis limitadas por un cierre estomático podrían provocar un daño irreversible en el aparato fotosintético por alta radiación. Bajo estas condiciones, en que el cloroplasto está sometido a un exceso de luz, se presentaría una regulación en la fotosíntesis en que intervienen las xantofilas disipando el exceso de energía, alcanzándose la protección y evitando el daño en los FSII (Demming-Adams 1990). Estos mecanismos de fotoprotección serían de una importancia mayor para la sobrevivencia en ambientes donde la planta está sometida a períodos prolongados de estrés ambiental (Ball et al. 1994).

En las especies de climas mediterráneos, la sequía, la radiación y las temperaturas en el verano o en el invierno, son todos factores que podrían directamente limitar la asimilación de $\mathrm{CO}_{2}$. A pesar de que en estos ecosistemas son escasos los estudios tendientes a comprender las implicaciones ecológicas del efecto interactivo de múltiples tipos de estrés, algunas investigaciones han sido realizadas con el propósito de comprender las respuestas a largo plazo en la fotosíntesis, en la eficiencia fotoquímica del FSII y en los mecanismos o sistemas de fotoprotección en las especies de zonas con clima tipo mediterráneo.

Estudios en Quercus ilex L. frente a la exposición a una elevada concentración de $\mathrm{CO}_{2}$ sometido a diferentes niveles de estrés hídrico (ScarasciaMugnozza et al. 1996) y en $Q$. suber, en relación a los cambios diurnos en los mecanismos de fotoprotección en esta especie, sugieren que aunque el cierre de los estomas explica la mayor parte de la variación en la asimilación de carbono a lo largo del verano, existe una disminución en el rendimiento fotoquímico del FSII como resultado del exceso de luz solar interceptada (Faria et al. 1998). Esto, precisamente, cuando la fotosíntesis es limitada por el cierre estomático debido al alto déficit de presión de vapor y/o de agua en el suelo. Además, se encontró que la conversión de viola hacia antera y zeaxantina, estaba correlacionada con la disipación térmica del exceso de energía lumínica absorbida. Estos cambios, sugieren, un alto grado de coordinación entre el comportamiento estomático, la capacidad fotosintética y los mecanismos de fotoprotección (Faria et al. 1998). Este efecto de la fotoinhibición en la ganancia total de carbono en las plantas, ha sido estimado por medio de modelos. Simulaciones realizadas en Quercus coccifera, una especie siempreverde, bajo condiciones climáticas que producen una fotoinhibición moderada, muestran una pérdida de la ganancia potencial de carbono de ca. $10 \%$ en capas del dosel expuestas al sol (Werner et al. 2001). En $Q$. suber y $Q$. ilex han sido descritos cambios estacionales en la composición de pigmentos del ciclo de las xantofilas en las hojas de sol y de sombra, encontrándose una mayor concentración al final del verano y en invierno, cuando la fotosíntesis está limitada por el estrés hídrico y el congelamiento, respectivamente (García-Plazaola et al. 1997). Además de encontrar una relación en ambos tipos de hojas entre estos pigmentos y el "quenching" o consumo no-fotoquímico, en $Q$. suber y en $Q$. ilex han sido descritos cambios diurnos en la composición de antioxidantes y carotenoides durante el verano o el invierno, respectivamente (Faria et al. 1996, García-Plazaola et al. 1999a).

El aumento del contenido de zeaxantina durante el período del día de mayor radiación bajo sequía, fue descrito inicialmente en las hojas de Arbutus unedo L., un arbusto esclerófilo, junto a la disminución reversible en la eficiencia fotoquímica del FSII relacionada con la disminu- 
ción en la asimilación de $\mathrm{CO}_{2}$ a medio día (Demmig-Adams et al. 1989). Estos, son similares a los cambios diurnos en el comportamiento de los estomas, de la fotosíntesis y los mecanismos de fotoprotección basados en la actividad de enzimas antioxidantes (superóxido dismutasa y ascorbato peroxidasa) en las hojas de Quercus suber (Faria et al. 1996). Un estudio comparativo sobre los cambios inducidos, tanto en primavera como en el verano, en el intercambio gaseoso y en la fotobiología de $Q$. ilex, árbol mediterráneo siempreverde, muestra una similar disminución en la fotosíntesis y en la conductancia estomática relacionada con el estrés hídrico (Penuelas et al. 1998). En California, así como en Heteromeles arbutifolia, un arbusto del chaparral, las altas temperaturas foliares en el verano son el factor limitante para la sobrevivencia en los juveniles de sombra que carecen de la fotoprotección estructural otorgada por los ángulos foliares (Valladares \& Percy 1997), el reclutamiento en $Q$. douglasii es limitado por la disponibilidad de agua en el suelo (Gordon \& Rice 2000). En las plántulas, una similar vulnerabilidad a la fotoinhibición inducida por temperatura o sequía en el límite arbóreo -con reducción en la ganancia de carbono asociada a una fotoinhibición crónica por carencia de recursos almacenados- explicaría, en algunas especies, la limitada capacidad de regeneración en condiciones extremas (Ball et al. 1991).

En la alta montaña, bajo condiciones ambientales extremas con una corta estación de crecimiento, las plantas requieren una asimilación eficiente de carbono (Körner \& Larcher 1988) cuando las bajas temperaturas nocturnas y los altos niveles de radiación en el verano alteran la capacidad fotosintética de las especies (Hälgren et al. 1990, Welander et al. 1994). Excepto por investigaciones en especies de alta montaña en Los Alpes (Lütz 1996, Streb et al. 1997) no he encontrado trabajos en que se describa el impacto de la fotoinhibición en el balance de carbono y/o la importancia de la fotoprotección en plantas de ambientes de alta montaña en zonas con clima de tipo mediterráneo. En estos, las plantas se podrían encontrar sometidas a condiciones extremas de temperatura, sequía y radiación, todos factores que favorecen y predisponen a las plantas a la fotoinhibición. Recientemente, sólo ha sido descrito el efecto de los micrositios, las formas de vida de las plantas y de las bajas temperaturas en la fotoinhibición en especies alpinas (Manuel et al. 1999) y en las montañas Rocosas (Germino \& Smith 2000). Las consecuencias de una disminución en la actividad fotosintética en plantas de ambientes extremos es un área de interés en los ecosistemas de alta montaña con clima tipo mediterráneo, debido a las limitaciones estacionales (sequía, temperaturas y luz) tanto en el crecimiento en adultos como para el establecimiento en los juveniles de las especies.

Königer et al. (1995) determinaron un mayor contenido de pigmentos de xantofilas en hojas de sol en especies del dosel y más bajos en plantas del sotobosque. Plantas en claros presentan valores intermedios en la fotosíntesis y contenido de pigmentos menor que los del dosel, patrón que explica diferencias en el nivel de fotoinhibición y capacidad de recuperación que presentarían las especies en ambientes con diferente nivel de luz (Oqüist et al. 1992, Lovelock et al. 1994). Es decir, debido a que la fotoprotección es un proceso de disipación de energía mediado por el ciclo de las xantofilas, la aclimatación y la tolerancia a altos niveles de luz estarían asociadas a una mayor concentración de estos pigmentos. Publicaciones en macroalgas, musgos, helechos, cactus y árboles confirman la importancia de la disipación térmica de la energía y su relación con estos pigmentos (Eickmeier et al. 1993, Franklin et al. 1996, Baker \& Adams 1997, Scholes et al. 1997, Deltoro et al. 1999).

Una especie semidecidua (Kageneckia angustifolia) en niveles altos de radiación en el verano, presenta diariamente un mayor y más rápido incremento en el nivel de disipación de energía en la antena colectora de luz, mostrando una rápida disminución en $\mathrm{F}_{\mathrm{V}}$ '/ $\mathrm{F}_{\mathrm{M}}$ ' y alto potencial fotoprotectivo (concentración de pigmentos) para la disipación de energía. Cuando las temperaturas nocturnas son congelantes o frente a la sequía de verano, con altos niveles de radiación y las altas temperaturas diurnas, la especie no presentan una fotoinhibición crónica de la fotosíntesis (H.M. Cabrera resultados no publicados). Sin embargo, esta combinación de condiciones podría explicar el límite altitudinal superior absoluto (ca. $2.200 \mathrm{~m}$ ) alcanzado por K. angustifolia en Chile central. Así mismo, en especies de alta montaña en zonas de clima tipo mediterráneo, el menor límite altitudinal del arbusto Anarthropyllum cumingii en comparación al de $A$. gayanum, se debería a su mayor sensibilidad al déficit hídrico estacional y a las bajas temperaturas (H.M. Cabrera resultados no publicados). Una menor sensibilidad a sufrir fotoinhibición por luz en exceso y la mayor tolerancia a la sequía, estaría relacionado a diferencias entre las especies tanto en la capacidad de utilización de la luz por la fotosíntesis, en la capacidad de disipación de energía por pigmentos del ciclo de las xantofilas, así como por un adecuado balance hídrico en las 
plantas. Las mayores tasas fotosintéticas, niveles de fotoprotección (concentración de pigmentos) y mecanismos de transporte-conservación de agua se encontrarían en las especies que siendo semideciduas ( $K$. angustifolia) y arbustivas ( $A$. cummingii) estarían por períodos mayores expuestas a sequía, alta radiación y altas temperaturas en los ecosistemas con clima tipo mediterráneo.

\section{AGRADECIMIENTOS}

Proyecto de Fortalecimiento de la Formación Inicial de Docentes, Vicerrectoría de Asuntos Docentes y Estudiantiles, Universidad Católica de Valparaíso. Fondos de instalación a exbecarios de la Red Latinoamericana de Botánica. Dra. Mary T. Kalin-Arroyo de la Universidad de Chile, Directora del Núcleo Milenio CMEB e Investigador Patrocinante del Proyecto Posdoctoral FONDECYT 3990039 del autor. Vicerrectoría de Investigación y Estudios Avanzados, Dirección de Investigación, Universidad Católica de Valparaíso, proyectos 202.780/1999, 122.755/ $2000,122.763 / 2001$ y $122.772 / 2002$. Al Sr. Félix López e Inmobiliaria DEZA y Compañía por fondos para implementar con equipos de investigación el Laboratorio de Fisiología y Ecofisiología Vegetal del autor en el Instituto de Biología de la Universidad Católica de Valparaíso.

\section{LITERATURA CITADA}

AL-MUFTI MM, CL SYDES, SB FURNESS, JP GRIME \& SR BAND (1977) A quantitative analysis of shoot phenology and dominance in herbaceous vegetation. Journal of Ecology 65: 759-791.

ALLEN DJ \& RO DONALD (2001) Impacts of chilling temperatures on photosynthesis in warm-climate plants. Trends in Plant Science 6: 36-41.

AGATI G, P MAZZINGHI, F FUSI \& I AMBROSINI (1995) The F685/F730 chlorophyll fluorescence ratio as a tool in plant physiology: response to physiological and environmental factors. Journal of Plant Physiology 145: 228-238.

ARMESTO JJ \& J MARTÍNEZ (1978) Relations between vegetation structure and slope aspect in the mediterranean region of Chile. Journal of Ecology 66: 881-896.

ARMESTO JJ, JR GUTIÉRREZ \& JA MARTÍNEZ (1979) Las comunidades vegetales de la región mediterránea de Chile: distribución de especies y formas de vida en un gradiente de aridez. Medio Ambiente (Chile) 4: 62-70.

ARO E-M, I VIRGIN \& B ANDERSON (1993) Photoinhibition of photosystem II. Inactivation, protein damage and turnover. Biochimica et Biophysica Acta 1143: 113-134.
ARROYO MTK, JJ ARMESTO \& C VILLAGRÁN (1981) Plant phenological patterns in the high Andean cordi1lera of central Chile. Journal of Ecology 69: 205223.

ARROYO MTK, JJ ARMESTO \& RB PRIMACK (1982). Community studies in pollination ecology in the high temperate Andes of central Chile, II. Effect of temperature on visitation rates and pollination possibilities. Plant Systematics and Evolution 149: 187-203.

ARROYO MTK \& P USLAR (1993) Breeding systems in a temperate mediterranean-type climate montane sclerophyllous forest in central Chile. Botanical Journal of the Linnean Society 111: 83-102.

ARROYO MTK, L CAVIERES, C MARTICORENA, \& M MUÑOZ (1995) Convergence in the mediterranean floras in central Chile and California: insights from comparative biogeography. En: Arroyo MTK, PH Zedler \& MD Fox (eds) Ecology and biogeography of Mediterranean ecosystems in Chile, California, and Australia: 43-88. Springer-Verlag, New York, New York.

BAKER NR (1991) A possible role for photosystem II in environmental perturbations of photosynthesis. Physiologia Plantarum 81: 563-570.

BAKER NR (1993) Light-use efficiency and photoinhibition of photosynthesis in plants under environmental stress. En: Smith JAC \& H Griffiths (eds) Water deficits plant responses from cell to community: 221-236. BIOS Scientific Publishers Limited, Oxford, United Kingdom.

BAKER DH \& WW ADAMS III (1997) The xanthophyll cycle and energy dissipation in differently oriented faced of the cactus Opuntia macrorrhiza. Oecologia 109: 352-361.

BALL MC, VS HODGES \& GP LAUGHLIN (1991) Coldinduced photoinhibition limits regeneration of snow gum at tree line. Functional Ecology 5: 663-668.

BALL MC, JA BUTTERWORTH, JS RODEN, R CHRISTIAN \& JJG EGERTON (1994) Application of chlorophyll fluorescence to forest ecology. Australian Journal of Plant Physiology 22: 311-319.

BELCHER JW, PA KEDDY \& L TWOLAN-STRUU (1995) Root and shoot competition intensity along a soil depth gradient. Journal of Ecology 83: 673-682.

BERENDSE F (1981) Competition between plant populations with different rooting depths. II. Pot experiments. Oecologia 48: 334-341.

BERENDSE F (1982) Competition between plant populations with different rooting depths. III. Field experiments. Oecologia 53: 50-55.

BILGER W \& O BJÖRKMAN (1994) Relationships among violaxanthin deepoxidation, thylakoid membrane conformation, and nonphotochemical chlorophyll fluorescence quenching in leaves of cotton (Gossypium hirsutum L.). Planta 193: 238-246.

BJÖRKMAN O \& B DEMING-ADAMS (1994) Regulation of photosynthetic light energy capture, convertion, and dissipation in leaves of higher plants. En: Schulze DE \& MM Caldwell (eds) Ecophysiology of photosynthesis: 17-45. Springer-Verlag, Berlin, Germany. 
CABRERA HM (1996) Temperaturas bajas y límites altitudinales en ecosistemas de plantas superiores: respuestas de las especies al frío en montañas tropicales y subtropicales. Revista Chilena de Historia Natural 69: 309-320.

CABRERA HM, F RADA \& L CAVIERES (1998) Effects of temperature on photosynthesis of two morphologically contrasting plant species along an altitudinal gradient in the tropical high Andes. Oecologia 114: 145-152.

CANADELL J \& PH ZEDLER (1995) Underground structures of woody plants in mediterranean ecosystems of Australia, California, and Chile. En: Arroyo MTK, PH Zedler \& MD Fox (eds) Ecology and biogeography of Mediterranean ecosystems in Chile, California, and Australia: 177-210. SpringerVerlag, New York, New York.

CAVIERES LA, F RADA, A AZÓCAR, C GARCÍANÚÑEZ \& HM CABRERA (2000) Gas exchange and low temperature resistance in two tropical high mountain tree species from the Venezuelan Andes. Acta Oecologica 21: 203-211

CODY ML \& HA MOONEY (1978) Convergence vs. nonconvergence in Mediterranean-climate ecosystems. Annual Review of Ecology and Systematics 9: 265-321.

COOMES DA \& PJ GRUBB (2000) Impacts of root competition in forests and woodlands: A theoretical framework and review of experiments. Ecological Monographs 70: 171-207.

CORNIC G (1994) Drought stress and high light effects on leaf photosynthesis. En: Baker NR \& JR Boeyer (eds) Photoinhibition of photosynthesis: from molecular mechanims to the field: 297-314. BIOS Scientific Publishers Limited, Oxford, United Kingdom.

COWLING RM, PH RUNDEL, BB LAMONT, MTK ARROYO \& M ARIANOUTSOU (1996) Plant diversity in mediterranean-climate regions. Trends in Ecology and Evolution 11: 362-366.

CHABOT BF \& WD BILLINGS (1972) Origins and ecology of the sierran alpine flora and vegetation. Ecological Monographs 42: 163-199.

DAMESIN C, S RAMBAL \& R JOFFRE (1997) Betweentree variations in leaf delta-13C of Quercus pubescens and Quercus ilex among Mediterranean habitats with different water availability. Oecologia 111: 26-35.

DELTORO VI, A CATALAYUD, F MORALES, A ABADIA \& E BARRENO (1999) Changes in the net photosynthesis, chlorophyll fluorescence and xanthophylls cycle interconversions during freezethaw cycles in the Mediterranean moss Leucodon sciuroides. Oecologia 120: 499-505.

DEMMING-ADAMS B (1990) Carotenoids and photoprotection in plants: a role for the xanthophyll zeaxanthin. Biochimica et Biophysica Acta 1020: 124.

DEMMIG-ADAMS B, WW ADAMS III, K WINTER, A MEYER, U SCHREIBER, JS PEREIRA, A KRÜGER, F-C CZYGAN \& OL LANGE (1989) Photochemical efficiency of photosystem II, photon yield of $\mathrm{O}_{2}$ evolution, photosynthetic capacity, and carotenoid composition during the midday depression of net $\mathrm{O}_{2}$ uptake in Arbutus unedo growing in Portugal. Planta 177: 377-387.
DEMMIG-ADAMS B \& WW ADAMS III (1992) Photoprotection and other responses of plants to high light stress. Annual Review of Plant Physiology and Plant Molecular Biology 43: 599-626.

DEMMIG-ADAMS B, WW ADAMS III, BA LOGAN \& AS VERHOEVEN (1995) Xanthophyll cycledependent energy dissipation and flexible photosystem II efficiency in plants acclimated to light stress. Australian Journal of Plant Physiology 22: 249-260.

DEMMIG-ADAMS B, WW ADAMS III, DH BARKER, BA LOGAN, DR BOWLING \& AS VERHOEVEN (1996) Using chlorophyll fluorescence to assess the fraction of absorbed light allocated to thermal dissipation of excess excitation. Physiologia Plantarum 98: 253-264.

DEKKER JP \& R VAN GRONDOLLE (2000) Primary charge separation in photosystem II. Photosynthesis Research 63: 195-208.

DI CASTRI F (1973) Climatographical comparisons between Chile and the western coast of North America. En: di Castri F \& HA Mooney (eds) Mediterraneantype ecosystems: origin and structure: 21-36. SpringerVerlag, Berlin, Germany.

DI CASTRI F (1990) An ecological overview of the five regions of the world with mediterranean climate. En: Groves RH \& F di Castri (eds) Biogeography of mediterranean invasions: 3-15. Cambridge University Press, Cambridge, United Kingdom.

EICKMEIER WG, C CASPER \& CB OSMOND (1993) Chlorophyll fluorescence in the resurrection plant Selaginela lepidophylla (Hook. \& Grev.) Spring during high-light and desiccation stress, and evidence for zeaxanthin-associated photoprotection. Planta 189: 3038.

FARIA T, JI GARCÍA-PLAZAOLA, A ABADIA, S CERASOLI, JS PEREIRA \& MM CHAVES (1996) Diurnal changes in photoprotective mechanisms in leaves of cork oak (Quercus suber) during summer. Tree Physiology 16: 115-123.

FARIA T, D SILVERIO, E BREIA, R CABRAL, A ABADIA, JS PEREIRA \& MM CHAVES (1998) Differences in the response of carbon assimilation to summer stress (water deficits, high light and temperature) in four Mediterranean tree species. Physiologia Plantarum 102: 419-428.

FRANKLIN LA, GGR SEATON, CE LOVELOCK \& AWD LARKUM (1996) Photoinhibition of photosynthesis on a coral reef. Plant Cell and Environment 19: 825836.

GARCÍA-PLAZAOLA JI, T FARIA, J ABADIA, A ABADIA, MM CHAVES MM \& JS PEREIRA (1997) Seasonal changes in xantophyll composition and photosynthesis of cork oak (Quercus suber L.) leaves under Mediterranean climate. Journal of Experimental Botany 48: 1667-1674.

GARCÍA-PLAZAOLA JI, U ARTETXE U \& MK DUNABITIA (1999a) Role of photoprotective systems of holm-oak (Quercus ilex) in the adaptation to winter conditions. Journal of Plant Physiology 155: 625-630.

GARCÍA-PLAZAOLA JI, U ARTETXE U \& JM BECERRIL (1999b) Diurnal changes in antioxidant and carotenoid composition in the Mediterranean schlerophyll tree Quercus ilex (L) during winter. Plant Science 143: 125-133. 
GERMINO MJ \& WK SMITH (2000) Differences in microsite, plant form, and low-temperature photoinhibition in alpine plants. Arctic, Antarctic and Alpine Research 32: 388-396.

GILIBERTO J \& H ESTAY (1978) Seasonal water stress in some Chilean matorral shrubs. Botanical Gazzete 139: 236-260.

GORDON DR \& KJ RICE (2000) Competitive suppression of Quercus douglasii (Fagaceae) seedling emergence and growth. American Journal of Botany 87: 986-994.

GRACE J (1993) Consequences of xilem cavitation for plant water deficits. En: Smith JAC \& H Griffiths (eds) Water deficits plant responses from cell to community: 109-128. BIOS Scientific Publishers Limited, Oxford, United Kingdom.

HÄLGREN J-E, T LUNDMARK \& M STRAND (1990) Photosynthesis of Sctos pine in the field after night frosts during summer. Plant Physiology and Biochemistry 28: 437-445.

HOFFMANN AJ \& MJ WALKER (1980) Growth habits and phenology of drought - deciduous species in an altitudinal gradient. Canadian Journal of Botany 58 : 1789-1796.

HOFFMANN AJ \& AE HOFMANN (1982) Altitudinal ranges of phanerophytes and chamaephytes in central Chile. Vegetatio 48: 151-163.

HOOK PB, WK LAUENROTH \& IC BURKE (1994) Spatial patterns of roots in a semiarid grassland: abundance of canopy openings and regeneration gaps. Journal of Ecology 82: 485-494.

HORTON P, AV RUBAN \& RG WALTERS (1994) Regulation of light harvesting in green plants: indication by nonphotochemical quenching of chlorophyll fluorescence. Plant Physiology 106: 415-420.

JARBEAU JA, FW EWERS \& SD DAVIS (1995) The mechanism of water stress-induced embolism in two species of chaparral shrubs. Plant Cell Environment 18: 189-196.

KOLB KJ \& SD DAVIS (1994) Drought-induced xylem embolism in co-occurring species of coastal sage and chaparral of California. Ecology 75: 648-659.

KÖNIGER M, GC HARRIS, A VIRGO \& K WINTER (1995) Xanthophyll-cycle pigments and photosynthetic capacity in tropical forest species: a comparative field study on canopy, gap and understory plants. Oecologia 104: 280-290.

KÖRNER CH \& W LARCHER (1988) Plant life in cold climates. En: Long SF \& FI Woodward (eds) Plants and temperature: 25-57. The Company of Biologists, Cambridge, United Kingdom.

KRAUSE GH \& E WEIS (1991) Chlorophyll fluorescence and photosynthesis: the basics. Annual Review of Plant Physiology and Plant Molecular Biology 42: 313-349.

KUMMEROW J (1981) Structure of roots and root systems. En: di Castri F, DW Goodall \& RL Specht (eds) Mediterranean-type shrublans: 269-288. Elsevier Scientific Publisher, Amsterdam, The Netherlands.

LAMONT BB (1995) Mineral nutrient relations in mediterranean regions of California, Chile and Australia. En: Arroyo MTK, PH Zedler \& MD Fox (eds) Ecology and biogeography of Mediterranean ecosystems in Chile, California, and Australia: 211238. Springer-Verlag, New York, New York.
LANGAN SJ, FW EWERS \& SD DAVIS (1997) Xylem dysfunction caused by water stress and freezing in two species of co-ocurring chaparral shrubs. Plant Cell Environment 20: 425-437.

LOGULlO MA \& S SALLEO (1993) Different vulnerabilities of Quercus ilex L. to freeze- and summer drought-induced xylem embolism: an ecological interpretation. Plant Cell Environment 16: 511-519.

LONG SP, S HUMPHRIES \& PG FALKOWSKI (1994) Photoinhibition of photosynthesis in nature. Annual Review of Plant Physiology and Plant Molecular Biology 45: 633-662.

LOVELOCK CE, M JEBB \& CB OSMOND (1994) Photoinhibition and recovery in tropical plant species: response to disturbance. Oecologia 97: 297-307.

LUTZ C (1996) Avoidance of photoinhibition and examples of photodestruction in high alpine Eriophorum. Journal of Plant Physiology 148: 120-128.

MANUEL N, G CORNIC, S AUBERT, P CHOLER, R BLIGNY \& U HEBER (1999) Protection against photoinhibition in the alpine plant Geum montanum. Oecologia 119: 149-158.

MARTÍNEZ JA \& JJ ARMESTO (1983) Ecophysiological plasticity and habitat distribution in three evergreen sclerophyllous shrubs of the Chilean matorral. Oecologia Plantarum 4: 211-219.

MELIS A (1999) Photosystem-II damage and repair cycle in chloroplasts: what modulates the rate of photodamage in vivo? Trends in Plant Science 4: 130-135.

MONTENEGRO G (1987) Quantification of mediterranean plant phenology and growth. En: Tenhunen JD, O Catarino, L Lange \& WC Oechel (eds) Plant response to stress: functional analysis in Mediterranean ecosystems: 469-488. Springer-Verlag, Berlin, Germany.

MONTENEGRO G \& R GINOCCHIO (1995) Ecomorphological characters as a resource for illustrating growth-form convergence in matorral, chaparral and mallee. En: Arroyo MTK, PH Zedler \& MD Fox (eds) Ecology and biogeography of Mediterranean ecosystems in Chile, California, and Australia: 160176. Springer-Verlag, New York.

MOONEY HA \& EL DUNN (1970) Photosynthetic systems of mediterranean-climate shrubs and trees of California and Chile. American Naturalist 104: 447453.

MOONEY HA \& J KUMMEROW (1971) The comparative water economy of representative evergreen sclerophyll and drought deciduous shrubs of Chile. Botanical Gazzete 132: 245-252.

MOONEY HA \& C CHU (1974) Seasonal carbon allocation in Heteromeles arbutifolia, a Californian evergreen shrub. Oecologia 14: 295-306.

MOONEY HA, AT HARRISON \& P MORROW (1975) Environmental limitations of photosynthesis on a Californian evergreen shrub. Oecologia 19: 203-301.

MOONEY HA, J KUMMEROW, AW JOHNSON, DJ PARSONS, S KEELEY, A HOFFMANN, RI HAYS, J GILIBERTO \& C CHU (1977) The producers - their resources and adaptative responces. En: Mooney HA (ed) Convergent evolution in Chile and California: Mediterranean climate ecosystems: 85-153. Dowden, Hutchinson and Ross, Stroudsburg, Pennsylvania. 
MOONEY HA (1983) Carbon-gaining capacity and allocation patterns of mediterranean-climate plants. En: Kruger FJ, DT Mitchell \& JUM Jarvis (eds) Mediterranean-type ecosystems: the role of nutrients: 103-119. Springer-Verlag, Heidelberg, Germany.

MÜLLER P, LI XIAO-PING \& KK NIYOGI (2001) Nonphotochemical quenching: a response to excess light energy. Plant Physiology 125: 1558-1566.

NAHAL I (1981) The mediterranean climate from a biological viewpoint. En: di Castri F, DW Goodall \& RL Specht (eds) Mediterranean type shrublands: 5464. Elsevier Sciencific Publishing Company, Amsterdan, The Netherlands.

NARDINI A, MA LO GULLO \& S SALLEO (1999) Competitive strategies for water availability in two Mediterranean Quercus species. Plant Cell Environment 22: 109-116.

NARDINI A, S SALLEO, MA LO GULLO MA \& F PITT (2000) Different responses to drought and freeze stress of Quercus ilex L. growing along a latitudinal gradient. Plant Ecology 148: 139-147.

OLIVARES SP \& FA SQUEO (1999) Patrones fenológicos en especies arbustivas del desierto costero del nortecentro de Chile. Revista Chilena de Historia Natural 72: 353-370.

ÖQUIST G, JM ANDERSON, T MCCAFLERY \& WS CHOW (1992) Mechanistic differences in photoinhibition of sun and shade plants. Planta 188: 422-430.

OSMOND CB (1981) Photorespiration and photoinhibition: some implications for the energetics of photosynthesis. Biochimica et Biophysica Acta 639: 77-98.

OSMOND CB (1994) What is photoinhibition? Some insights from comparisons of shade and sun plants. En: Baker NR \& JR Bowyer (eds) Photoinhibition of photosynthesis: from molecular mechanisms to the field: 1-24. BIOS Scientific Publishers Limited, Oxford, United Kingdom.

PANTASTICO-CALDAS M \& DL VENABLE (1993) Competition in two species of desert annuals along a topographic gradient. Ecology 74: 2192-2203.

PARSONS DJ \& AR MOLDENKE (1975) Convergence in vegetation structure along analogous climatic gradients in California and Chile. Ecology 56: 950957.

PEARSON DJ (1976) Vegetation structure in mediterranean scrub communities of California and Chile. Journal of Ecology 64: 435-447.

PENUELAS J, I FILELLA, D SISCART \& J PINOL (1998) comparative field study of spring and summer leaf gas exchange and photobiology of the Mediterranean trees Quercus ilex and Phillyrea latifolia. Journal of Experimental Botany 49: 229-238.

PEREIRA JS \& MM CHAVEZ (1993) Plant water deficits in Mediterranean ecosystems. En: Smith JAC \& H Griffiths $\mathrm{H}$ (eds) Water deficits plant responses from cell to community: 221-236. BIOS Scientific Publishers Limited, Oxford.

PFUNDEL JS \& W BILGER (1994) Regulation of possible function of the violaxanthin cycle. Photosynthesis Research 42: 89-109.
PRENTICE IC, W CRAMER, SP HARRISON, R LEEMANS, RA MONSERUD \& A SOLOMON (1992) A global biome model based on plant physiology and dominance, soil properties and climate. Journal of Biogeography 19: 117-134.

RADA F, A AZÓCAR, B BRICEÑO, J GONZÁLEZ \& C GARCÍA-NÚÑEZ (1996) Carbon and water balance in Polylepis sericea, a tropical treeline species. Trees 10: 218-222.

RADA F, FA SQUEO, A AZÓCAR \& HM CABRERA (1999) Water and carbon relations in the genus Adesmia (Papilionaceace) at different altitudes in the high north-central Chilean Andes. Revista Chilena de Historia Natural 72: 201-211.

RADA F, GARCÍA-NÚÑEZ C \& C BOERO (2001) Lowtemperature resistance in Polylepis tarapacana, a tree growing at the highest altitudes in the world. Plant Cell and Environment 24: 377-381.

REDTFELDT RA \& SD DAVIS (1996) Physiological and morphological evidence of niche segregation between two co-occurring species of Adenostoma in California chaparral. Ecoscience 3: 290-296.

RIVEROS F, A HOFFMANN, G ÁVILA, ME ALJARO, S ARAYA, AE HOFFMANN \& G MONTENEGRO (1976) Comparative morphological and ecophysiological aspects of two sclerophyllous Chilean shrubs. Flora 165: 223-234.

ROYCE EB \& MG BARBOUR (2001a) Mediterranean climate effects. I. Conifer water use across a Sierra Nevada ecotone. American Journal of Botany 88: 911-918.

ROYCE EB \& MG BARBOUR (2001b) Mediterranean climate effects. II. Conifer growth phenology across a Sierra Nevada ecotone. American Journal of Botany 88: 911-918.

RUNDEL PW (1981) The matorral zone of central Chile. En: di Castri F, DW Goodall \& RL Specht (eds) Mediterranean type shrublands: 175-201. Elsevier Scientific Publishers, Amsterdam, The Netherlands.

SANTIBÁÑEZ F \& JM URIBE (1992) Atlas agroclimático de Chile: Región V y Metropolitana. Universidad de Chile, Facultad de Ciencias Agrarias y Forestales, Santiago.

SCARASCIA-MUGNOZA AG, A DE ANGELIS, G MATTEUCCI \& R VALENTINI (1996) Long-term exposure to elevated $\mathrm{CO}_{2}$ in a natural Quercus ilex $\mathrm{L}$. community: net photosynthesis and photochemical efficiency of PSII at different levels of water stress. Plant Cell and Environment 19: 643-654.

SCHOLES JD, MC PRESS \& SW ZIPPERLEN (1997) Differences in light energy utilization and dissipation between dipterocarp rain forest tree seedlings. Oecologia 109: 41-48.

SEATON GGR \& DA WALKER (1990) Chlorophyll fluorescence as a measure of photosynthetic carbon assimilation. Proceedings of the Royal Society of London B 242: 29-35.

SMITH WK \& RA DONAHUE (1991) Simulated influence of altitude on photosynthetic $\mathrm{CO}_{2}$ uptake potential in plants. Plant Cell Environment 14: 133-136.

SPERRY JS \& MT TYRRE (1988) Mechanism of water stress-induced xylem embolism. Plant Physiology 88: 581-587. 
SPERRY JS \& JEM SULLIVAN (1992) Xylem embolism in response to freeze-thaw cycles and water stress in ring-porous, diffuse-porous, and conifer species. Plant Physiology 100: 605-613.

SPERRY JS (1995) Limitations on stem water transport and their consequences. En: Gartner BL (ed) Plant stems: physiology and functional morphology: 105124. Academic Press, San Diego, California.

SQUEO FA, JR EHLERINGER, N OLIVARES \& G ARANCIO (1994) Variation in leaf level energy balance components of Encelia canescens along a precipitation gradient in north-central Chile. Revista Chilena de Historia Natural 67: 143-155.

SQUEO FA, F RADA, CE GARCÍA, AL PONCE, A ROJAS \& A AZÓCAR (1996) Cold resistance mechanisms in high desert Andean plants. Oecologia 105: 552-555.

SQUEO FA, N OLIVARES, S OLIVARES, A POLLASTRI, E AGUIRRE, R ARAVENA, C JORQUERA \& JR EHLERINGER (1999) Grupos funcionales en arbustos desérticos definidos en base a las fuentes de agua utilizadas. Gayana Botánica (Chile) 56: 1-15.

STREB P, J FEIERABEND \& R BLIGNY (1997) Resistance to photoinhibition of photosystem II and catalase and antioxidative protection in high mountain plants. Plant Cell and Environment 20: 1030-1040.

TENHUNEN JD, LO LANGE, J GEBEL, W BEYSCHLAG \& JA WEBER (1984) Changes in photosynthetic capacity, carboxilating efficiency, and $\mathrm{CO}_{2}$ compensation point associated with midday stomatal closure and midday depression of net $\mathrm{CO}_{2}$ exchange of leaves of Quercus suber. Planta 193: 193-203.
TOGNETTI R, A LONGOBUCCO, F MigLietTA \& A RASCHI (1998) Transpiration and stomatal behaviour of Quercus ilex plants during the summer in a Mediterranean carbon dioxide spring. Plant Cell Environment 21: 613-622.

TOGNETTI R, A MINNOCCI, J PENUELAS, A RASCHI \& MB JONES (2000) Comparative field water relations of three Mediterranean shrub species coocurring at a natural $\mathrm{CO}_{2}$ vent. Journal of Experimental Botany 51: 1135-1146.

VALLADARES F \& RW PEARCY (1997) Interactions between water stress, sun-shade acclimation, heat tolerance and photoinhibition in the sclerophyll Heteromeles arbutifolia. Oecologia 111: 505-514.

VERDAGUER D, PJ CASERO \& M MOLINAS (2000) Lateral root development in a woody plant, Quercus suber L. (cork oak). Canadian Journal of Botany 78 : 1125-1135.

WANG J, EN IVES \& MJ LECHOWICZ (1992) The relation of foliar phenology to xylem embolism in trees. Functional Ecology 6: 469-475.

WELANDER NT, P GEMMEL, O HELLGREN \& B OTTOSSON (1994) The consequences of freezing temperatures followed by high irradiance on in vivo chlorophyll fluorescence and growth in Picea abies. Physiologia Plantarum 91: 121-127.

WERNER C, RJ RYEL, O CORREIA O \& W BEYSCHLAG (2001) Effects of photoinhibition on whole-plant carbon gain with a photosynthesis model. Plant Cell and Environment 24: 27-40.

WOODWARD FI \& BG WILLIAMS (1987) Climate and plant distribution at global and local scales. Vegetatio 69: 189-197.

Editor Asociado: J. Gutiérrez

Recibido el 22 de noviembre de 2001; aceptado el 8 de julio de 2002 